\title{
Histone deacetylase inhibitor trichostatin A and autophagy inhibitor chloroquine synergistically exert anti-tumor activity in H-ras transformed breast epithelial cells
}

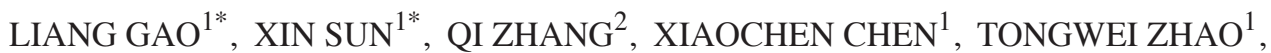 \\ LIQING LU ${ }^{1}$, JIANBIN ZHANG ${ }^{1}$ and YUPENG HONG ${ }^{1}$
}

${ }^{1}$ Department of Oncology, Zhejiang Provincial People's Hospital, People's Hospital of Hangzhou Medical College, Hangzhou, Zhejiang 310014; ${ }^{2}$ Department of Hepatobiliary and Pancreatic Surgery, The Second Affiliated Hospital, Zhejiang University School of Medicine, Hangzhou, Zhejiang 310002, P.R. China

Received July 10, 2017; Accepted January 12, 2018

DOI: $10.3892 / \mathrm{mmr} .2018 .8446$

\begin{abstract}
Histone deacetylase inhibitors (HDACIs) cause oncogene-transformed mammalian cell death. Our previous study indicated that HDACIs activate forkhead box O1 (FOXO1) and induce autophagy in liver and colon cancer cells. However, whether FOXO1 is involved in HDACI-mediated oncogene-transformed mammalian cell death remains unclear. In the present study, H-ras transformed MCF10A cells were used to investigate the role of FOXO1 in this pathway. Results showed that trichostatin A (TSA), a HDACI, activated apoptosis in MCF10A-ras cells, but not in MCF10A cells. Furthermore, TSA activated FOXO1 via P21 upregulation, whereas the knockdown of FOXO1 reduced TSA-induced cell death. In addition, TSA induced autophagy in MCF10A and MCF10A-ras cells by blocking the mammailian target of rapamycin signaling pathway. Furthermore, autophagy inhibition lead to higher MCF10A-ras cell death by TSA, thus indicating that autophagy is essential in cell survival. Taken together, the present study demonstrated that TSA causes oncogene-transformed cell apoptosis via activation of FOXO1
\end{abstract}

Correspondence to: Dr Yupeng Hong or Dr Jianbin Zhang, Department of Oncology, Zhejiang Provincial People's Hospital, People's Hospital of Hangzhou Medical College, 158 Shangtang Road, Hangzhou, Zhejiang 310014, P.R. China

E-mail: hypbdn@zju.edu.cn

E-mail: zhangjianbin@hmc.edu.cn

"Contributed equally

Abbreviations: CQ, chloroquine; FOXO1, forkhead box O1; HDACIs, histone deacetylase inhibitors; mTOR, mammailian target of rapamycin; PARP1, poly-ADP-ribose polymerase-1; TSA, trichostatin A

Key words: trichostatin A, histone deacetylase inhibitor, apoptosis, autophagy, MCF10A-ras cells, chloroquine and HDACI-mediated autophagy induction, which served as important cell survival mechanisms. Notably, the present findings imply that a combination of HDACIs and autophagy inhibitors produce a synergistic anticancer effect.

\section{Introduction}

Autophagy, an evolutionarily conserved cellular degradation pathway, has been shown to be adaptable to starvation and some other stress conditions (1). Autophagy plays a critical role in biological processes, including cell metabolism, survival, death and degradation, and recycling of cellular components. Additionally, autophagy is involved in the pathogenesis of essential diseases including neurodegenerative disease, metabolic disorders, and noteworthy cancer (2-5). The role of autophagy in cancer is one of a double-edged sword: While it renders tumor cells the ability to surmount metabolic stress, including hypoxia and insufficient nutrients, it can also inhibit cancer cells proliferation through oncogenic proteins degradation (3). In addition, autophagy in tumor cells is elicited during tumor progression to accommodate the metabolic stress (6). However, if the cancer cells cannot sustain such metabolic stress, autophagic cell death might occur, suggesting a new target for cancer therapy $(7,8)$; Many drugs, including mammalian target of rapamycin (mTOR) inhibitors, proteasome, and histone deacetylases, can induce autophagy, and are thus considered efficacious tools $(3,4)$.

Histone deacetylase inhibitors (HDACIs) are a class of promising target molecules for cancer treatment, which act through the regulation of the acetylation states of histone proteins and other non-histone protein targets. HDACIs induces cell cycle arrest, differentiation, apoptosis, and autophagy $(9,10)$. Generally, induction of apoptosis is essential for the antitumor activity of HADCIs (11). There is numerous evidence indicating that HDACIs can cause morphological alteration and cell cycle arrest in oncogene-transformed or tumor cells (12). Moreover, recent studies have exploited the antitumor effect of HDACIs including suberoylanilide hydroxamic acid (SAHA) and trichostatin A (TSA) through inducing autophagy (13-15). 
The forkhead box proteins (FOXOs) including FOXO1, FOXO3, FOXO4 and FOXO6 are essential for genes regulation (16). Among these proteins, the functions of FOXO1 like cell cycle arrest, apoptosis, defense against oxidative stress and DNA repair are the most clearly elucidated $(16,17)$. Recently, it has been reported that FOXO1 acetylation is involved in HDACIs-mediated autophagy (10). Nonetheless, the involvement of FOXO1 in HDACIs-caused oncogene-transformed mammalian cells death remains unclear and requires further investigation.

In the present study, we investigate the antitumor effect of TSA in H-ras-transformed human breast epithelial cells (MCF10A-ras cells) through a FOXO1-dependent pathway. In addition, we found that TSA could induce autophagy in MCF10A-ras cells through blocking mTOR pathway; such autophagy served as a pro-survival mechanism in TSA-mediated cell death. Finally, we found that combination of TSA and autophagy inhibitor chloroquine (CQ) exerted a synergistic antitumor effect.

\section{Materials and methods}

Cell cultures. MCF10A and MCF10A-ras cells were provided by Prof. Shen Hanming (National University of Singapore). All cells were maintained in DMEM (D1152; Sigma-Aldrich; Merck KGaA, Darmstadt, Germany) supplemented with 5\% horse serum, $0.5 \mu \mathrm{g} / \mathrm{ml}$ hydrocortisone, $10 \mu \mathrm{g} / \mathrm{ml}$ insulin, $20 \mathrm{ng} / \mathrm{ml}$ epidermal growth factor (EGF), $0.1 \mu \mathrm{g} / \mathrm{ml}$ cholera enterotoxin, $100 \mathrm{U} / \mathrm{ml}$ penicillin-streptomycin, $2.5 \mathrm{mM}$ L-glutamine and $0.5 \mu \mathrm{g} / \mathrm{ml}$ fungizone, in a humidified atmosphere containing $5 \% \mathrm{CO}_{2} / 95 \%$ air at $37^{\circ} \mathrm{C}$. The culture medium was replaced every 2 days.

Reagents and antibodies. The chemicals and reagents used in our experiments were purchased as follows: TSA (T8552; Sigma-Aldrich; Merck KGaA); CQ (C6628; Sigma-Aldrich; Merck KGaA); FOXO1 (2880; Cell Signaling Technology, Inc., Danvers, MA, USA); microtubule-associated protein1 light chain 3/LC3 (L7543; Sigma-Aldrich; Merck KGaA); poly-ADP-ribose polymerase-1 (PARP1; 9542; Cell Signaling Technology, Inc.); CDKN1A/P21 (2947; Cell Signaling Technology, Inc.); P62/SQSTM1 (P0067; Sigma-Aldrich; Merck KGaA); phospho-S6 (S235/236; 2211; Cell Signaling Technology, Inc.); phosphor-AKT (ser473; 4060; Cell Signaling Technology, Inc.); HSP70 (4872; Cell Signaling Technology, Inc.); PARP1 (9542; Cell Signaling Technology, Inc.); caspase-3 (9662; Cell Signaling Technology, Inc.); Cathepsin D (2284; Cell Signaling Technology, Inc.); and $\beta$-actin (A5441; Sigma-Aldrich; Merck KGaA).

Reverse transcription-quantitative PCR. RNA was extracted using RNeasy kit (217004; Qiagen GmbH, Hilden, Germany). A reverse transcription reaction was performed using $1 \mu \mathrm{g}$ of total RNA with High Capacity cDNA Reverse Transcription kit (4368814; Applied Biosystems; Thermo Fisher Scientific, Inc.), following the manufacturer's instructions. The mRNA expression levels were determined by qPCR using SsoFast ${ }^{\mathrm{TM}}$ EvaGreen Supermix (172-5201; Bio-Rad Laboratories, Inc., Hercules, CA, USA) and CFX96 Touch ${ }^{\mathrm{TM}}$ Real-Time PCR Detection System (Bio-Rad Laboratories, Inc.).
Glyceraldehyde-3-phosphate dehydrogenase (GAPDH) was used as an internal control of RNA integrity. qPCR was performed in triplicate.

Small interfering RNA (siRNA) and transient transfection. DharmaFECT 4 Transfection Reagent (T-2001-02; GE Healthcare Dharmacon, Inc., Lafayette, CO, USA) was used to transfected the scramble RNAi oligonucleotides and siRNAs targeting FOXO1 (6242; Cell Signaling Technology, Inc.) into MCF10A-ras cells according to manufacturer's instructions. After transfection, cell lysates were detected by western blotting.

Western blotting. The treated cells were lysed in Laemmli SDS buffer (62.5 mM Tris at pH 6.8) 25\% glycerol, $2 \%$ SDS, phosphatase inhibitor (78428; Pierce; Thermo Fisher Scientific, Inc.) and proteinase inhibitor cocktail (11836153001; Roche Applied Science, Madison, WI, USA). The cell lysates were boiled and then prepared for western blotting after lysis. Equal amount of proteins was resolved by SDS-PAGE and then transferred onto PVDF membrane. The membranes were probed with selected primary and secondary antibodies after blocking with 5\% nonfat milk, and then were developed using enhanced chemiluminescence method. Finally, the membranes were visualized using the Kodak Image Station 4000R (Kodak, Rochester, NY, USA).

Detection of viable and dead cells. Morphological changes under phase-contrast microscopy and propidium iodide (PI) live cell uptake assay, coupled with flow cytometry were used to quantitatively and qualitatively examine cell death. For PI staining, the medium in each plastic well was collected and cells were harvested with trypsin after treatments. Then, cells were resuspended in $1 \mathrm{x}$ phosphate-buffered saline containing PI at a final concentration of $5 \mu \mathrm{g} / \mathrm{ml}$ and incubated at $37^{\circ} \mathrm{C}$ for $10 \mathrm{~min} .10,000$ cells from each sample were analyzed through FACSCalibur flow cytometry (BD Biosciences, San Jose, CA, USA) using CellQuest software.

Statistical analysis. All western blotting and image data presented in this study are representatives from at least 3 independent experiments. All data are illustrated as the mean \pm standard deviation from triplicate independent experiments performed in a parallel manner and analyzed through the ANOVA followed by Dunnett's post hoc test. The statistical significance is indicated as $\mathrm{P}<0.05, \mathrm{P}<0.01$ and $\mathrm{P}<0.001$.

\section{Results}

Morphological changes and cell viability by TSA. As shown in Fig. 1A, MCF10A-ras cells changed their shape from rounded to spindle, indicating that an oncogenic transformation caused significant morphological alterations in these cells. After treatment with $0.5 \mu \mathrm{M}$ TSA for $24 \mathrm{~h}$, the morphology of MCF10A-ras cells dramatically shifted to an elongated shape with filamentous protrusions; while, no discernable changes were found in MCF10A cells (Fig. 1A). Furthermore, after treatment with $0.5 \mu \mathrm{M}$ TSA for $24 \mathrm{~h}$, significantly higher cell death percentage was observed in MCF10A-ras cells (Fig. 1B and C). 


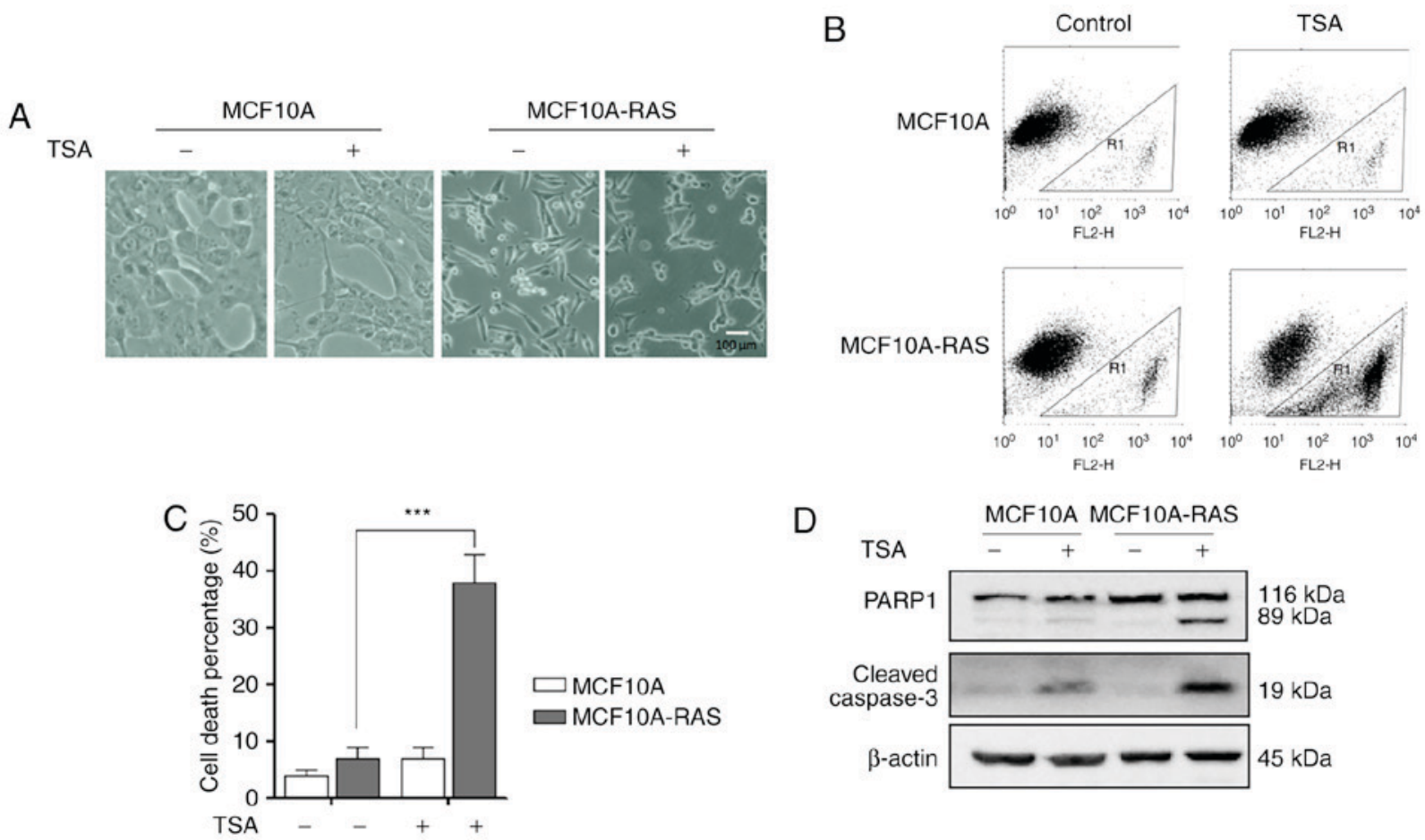

Figure 1. Effects of TSA on MCF10A-ras cells. (A) Morphological changes in the MCF10A and MCF10A-ras cells after treatment of TSA with concentration of $0.5 \mu \mathrm{M}$ for $24 \mathrm{~h}$. The cells morphology was examined under phage-contrast microscopy. Scale bar $100 \mu \mathrm{m}$. (B) Flow cytometry of MCF10A and MCF10A-ras cells treated with $0.5 \mu \mathrm{M}$ TSA for $24 \mathrm{~h}$. (C) MCF10A and MCF10A-ras cells were treated with $0.5 \mu \mathrm{M}$ TSA for $24 \mathrm{~h}$. The cell viability was measured using PI live cell uptake assay coupled with flow cytometry. Data are illustrated as the mean \pm SD $\left({ }^{* * *} \mathrm{P}<0.001\right)$. (D) Effect of TSA on the expression of cleaved PARP1 and Caspase-3. The proteins were extracted from MCF10A and MCF10A-ras cells treated with $0.5 \mu \mathrm{M}$ TSA for $24 \mathrm{~h}$. The expression of proteins was determined by western blotting analysis using the indicated antibody. $\beta$-actin was used as a loading control. TSA, trichostatin A; PI, propidium iodide; PARP1, poly-ADP-ribose polymerase-1.

TSA treatment causes MCF10A-ras cell apoptosis. The effects of TSA on the cleavage of PARP1 and Caspase-3 were examined to determine the underlying molecular mechanism of the TSA-induced cell death. As shown in Fig. 1D, TSA significantly elevated the levels of cleaved Caspase-3 and PARP1 in MCF10A-ras cells compared to MCF10A cells. These results demonstrated that TSA could induce MCF10A-ras cell apoptosis.

TSA treatment increases the activity of FOXO1. It was reported that FOXO1 is essential in regulating apoptosis and autophagy (16). Therefore, the possibility of involvement of FOXO1 in TSA-induced apoptosis was investigated. Firstly, we investigated the transcriptional level changes of FOXO1 in MCF10A and MCF10A-ras cells. As shown in Fig. 2A, we performed qPCR to measure the mRNA levels of FOXO1, and found that TSA treatment induced significant increase of FOXO1 mRNA level in MCF10A-ras cells compared to MCF10A cells. Secondly, TSA induced an increase in FOXO1, P21 and cleaved Caspase-3 expression n MCF10A-ras cell lines compared to MCF10A cells (Fig. 2B).

Furthermore, to confirm the role of FOXO1 in HDACIs TSA-mediated MCF10A-ras cell death, FOXO1 was silenced by siRNA. As expected, knockdown of FOXO1 markedly reduced the expression level of cleaved Caspase-3 and reduced cell death percentage in MCF10A-ras cells (Fig. 2C-E).

TSA treatment induces autophagy via blocking mTOR pathway. TSA can suppress cell proliferation and induce cell death through effective inhibition of HDAC enzyme activity at nanomolar concentrations (18). To investigate TSA's effect on autophagy, we treated MCF10A-ras cells with TSA. Briefly, our results indicated that TSA enhanced autophagy through increasing LC3, Cathepsin D and HSP70, and decreasing P62 in both MCF10A and MCF10A-ras cells. It has been reported that HDACIs can induce autophagy via downregulation of AKT-mTOR signaling (13). Therefore, we determined the role of mTOR pathway in autophagy induction by TSA. As shown in Fig. 3A, TSA reduced phospho-AKT and phosphor-S6 expression levels in both MCF10A and MCF10A-ras cells, suggesting the suppression of mTOR activity.

Suppression of autophagy sensitizes TSA-caused cell death. Previous results showed that TSA could induce autophagy in MCF10A-ras cells, hence we investigated whether the inhibition of autophagy would sensitize TSA-caused cell death. Briefly, we treated the tumor cells with a pharmacological inhibitor CQ, an inhibitor of the lysosomal $\mathrm{pH}$ gradient. Fig. 3B revealed the morphological changes with the treatment of combination of CQ and TSA. TSA treatment triggered more MCF10A-ras cell deaths in the presence of CQ (Fig. 3C, D), supporting the notion that TSA-induced autophagy served as a cell survival mechanism. This result was consistent with our previous studies (10).

\section{Discussion}

HDACIs are new antitumor agents, which exert a great influence on cancer cells promoting cell death, apoptosis, cell cycle 

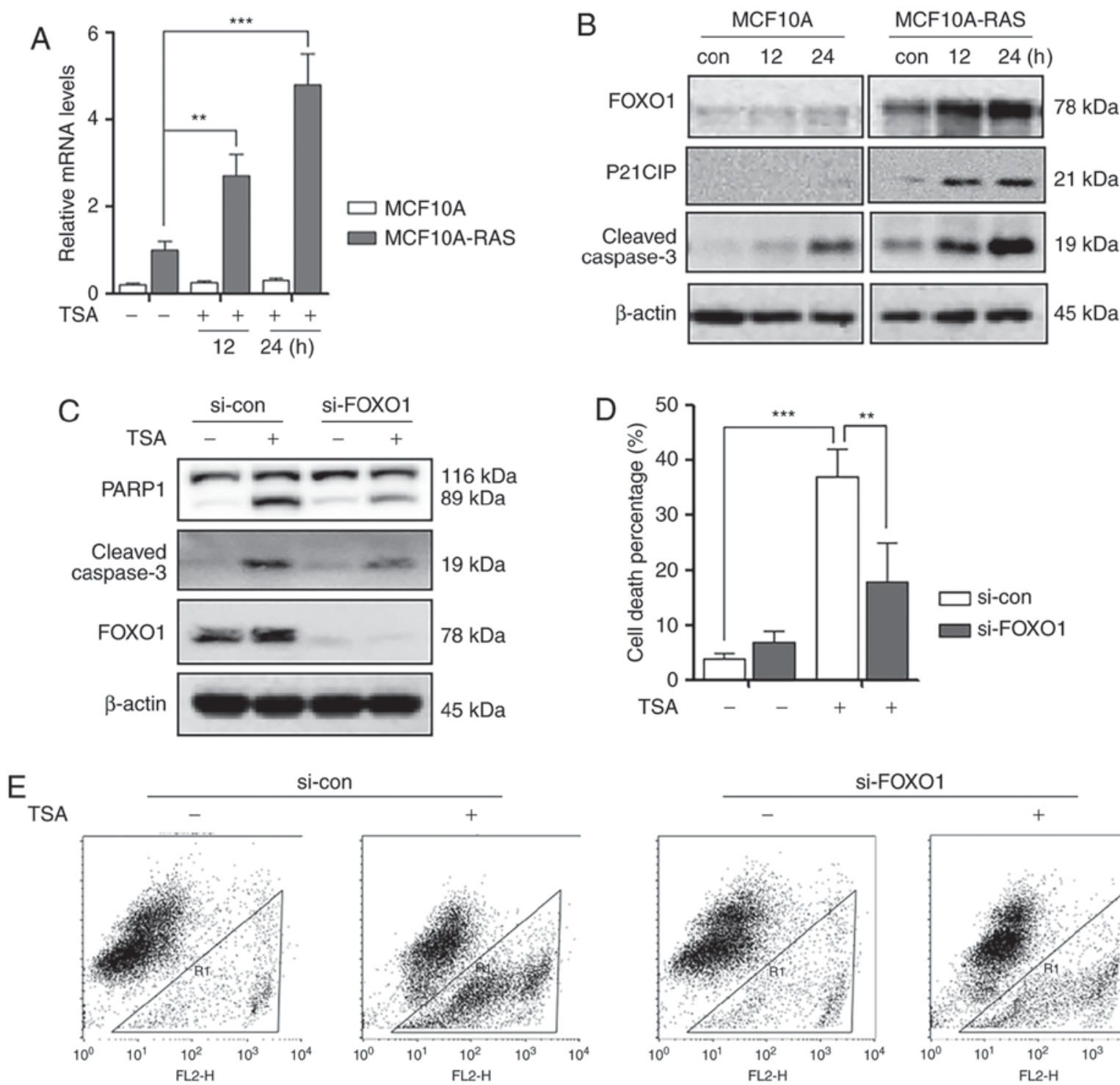

si-con
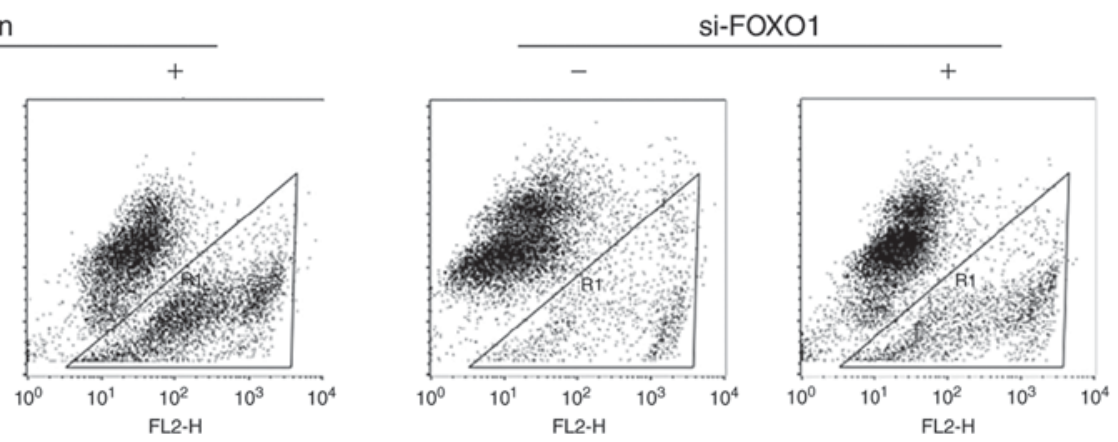

Figure 2. TSA treatment activates FOXO1 and causes MCF10A-ras cell death. (A) MCF10A and MCF10A-ras cells were treated with $0.5 \mu \mathrm{M}$ TSA for 12 or $24 \mathrm{~h}$. The cells were harvested for mRNA extraction and qPCR was performed to determine FOXO1 level. GAPDH was used as an internal control. "* P $<0.01$, ${ }^{* * * *} \mathrm{P}<0.001$. (B) MCF10A and MCF10A-ras cells were treated with $0.5 \mu \mathrm{M}$ TSA for 12 or $24 \mathrm{~h}$. The cells were harvested and subjected to western blotting analysis to evaluate FOXO1, P21 and cleaved Caspase-3 expression. $\beta$-actin was used as a loading control. (C) Control siRNA and FOXO1 siRNA were transfected into MCF10A-ras cells according the protocol. MCF10A-ras cells then were treated with $0.5 \mu \mathrm{M}$ TSA for $24 \mathrm{~h}$. Cells were harvested and immunoblotted for FOXO1, PARP1 and cleaved Caspase-3 antibodies. $\beta$-actin was used as a loading control. (D) MCF10A-ras cells were transiently transfected with control siRNA and FOXO1 siRNA according to the protocol and then were treated with $0.5 \mu \mathrm{M}$ TSA for $24 \mathrm{~h}$. The cell viability was measured using PI live cell uptake assay coupled with flow cytometry. Data are illustrated as the mean $\pm \mathrm{SD}$. ${ }^{* *} \mathrm{P}<0.01,{ }^{* * * *} \mathrm{P}<0.001$. (E) Flow cytometry of MCF10A-ras cells were transiently transfected with control siRNA and FOXO1 siRNA and then were treated with $0.5 \mu \mathrm{M}$ TSA for $24 \mathrm{~h}$. TSA, trichostatin A; FOXO1, forkhead box O1.

arrest and autophagy (9-11). In our study, TSA was found to induce apoptosis in MCF10A-ras cells through activation of FOXO1. In addition, siRNA knockdown of FOXO1 in MCF10A-ras cells markedly reduced TSA-induced apoptosis and dramatically attenuated the antitumor effect of TSA.

Deacetylase and acetyltransferase, mediating post-transcriptional regulation, are involved in the regulation of apoptosis (19). It has been shown that the HACIs can facilitate apoptosis though upregulation of pro-apoptotic gens and downregulation of anti-apoptotic genes (9). TSA, one of HDACIs, has been shown to significantly induce the apoptosis of MCF10A-ras cells by promoting expression of Bax (20). Our data were generally concordant with earlier reports suggesting that TSA was capable of inducing apoptosis in MCF10A-ras cells (20).
Furthermore, our study clearly showed that the FOXO1 played a critical role in TSA-induced apoptosis. A previous study has proved that HDACIs could induce apoptosis through the FOXO1-Bim pathway and that FOXO1 knockdown could protect from TSA-caused cell death $(10,21)$. In our study, TSA, one of HDACIs, concurrently increased the expression levels of FOXO1 in MCF10A-ras cells with the expression of cleavage of Caspase-3. Conversely, in the context of the knockdown of FOXO1, the expression of cleaved Caspase 3 was attenuated indicating that the apoptosis of MCF10A-ras cells induced by TSA was inhibited. As a result, the cell death was reduced in the MCF10A-ras cell. Overall, to the best of our knowledge this was the first study showing that TSA-induced apoptosis observed in MCF10A-ras cells was dependent on FOXO1 activity. 
A

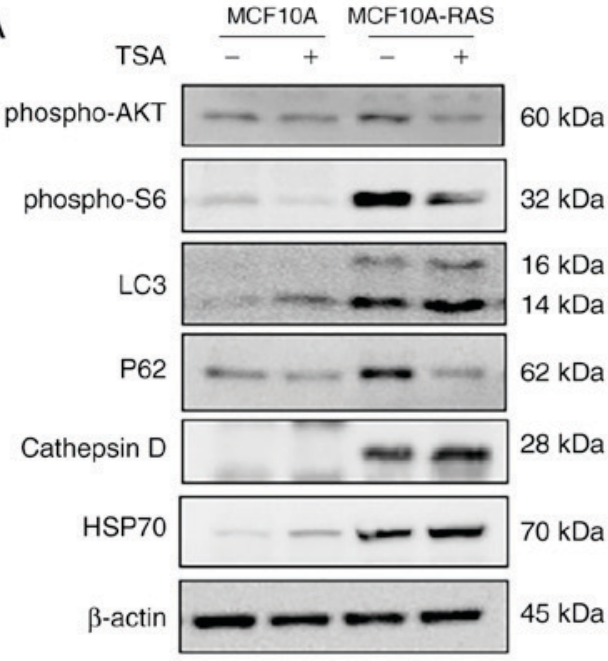

B

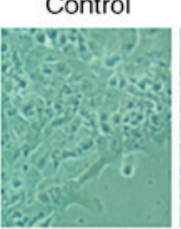

MCF10A-RAS

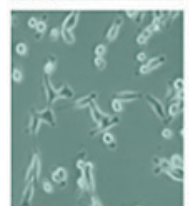

$C Q$

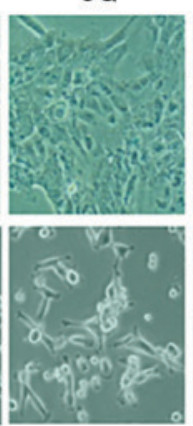

TSA

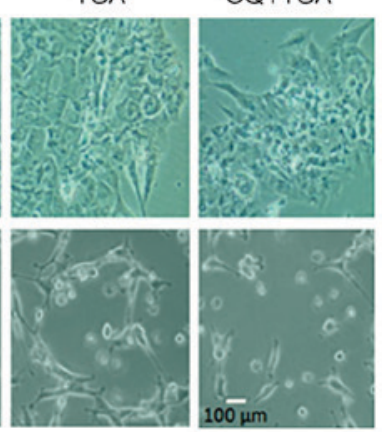

C

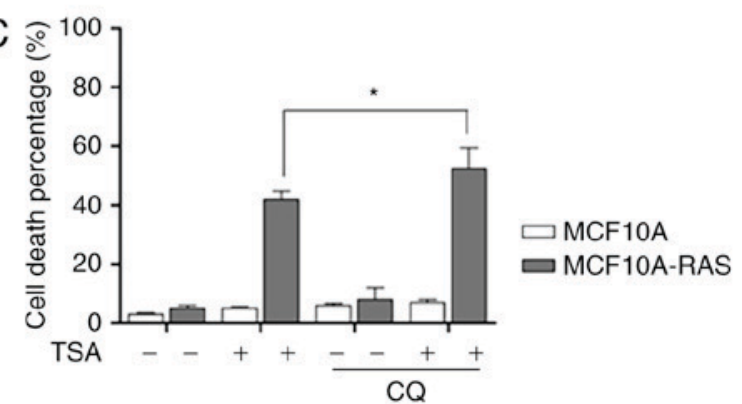

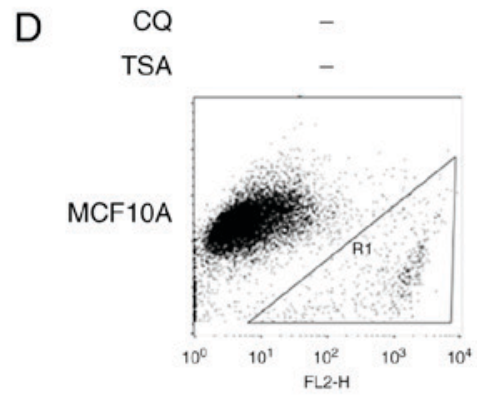

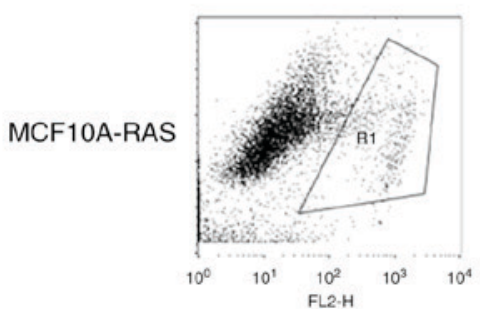

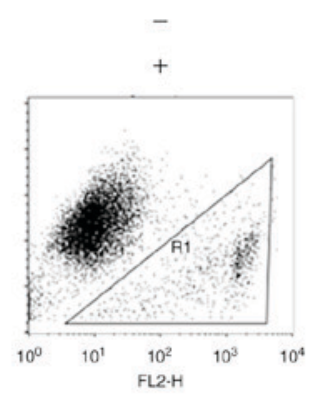

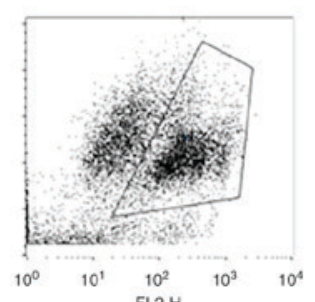

$+$
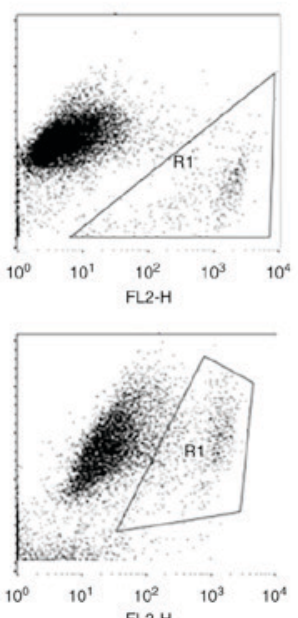

$+$

$+$
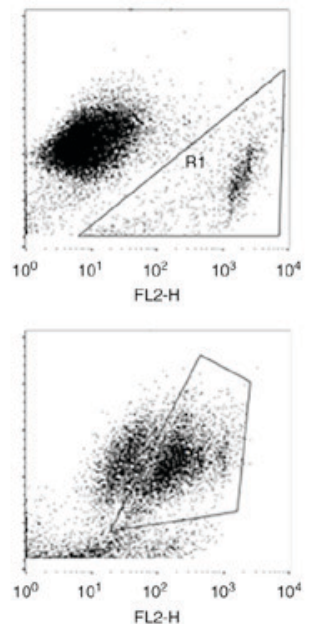

Figure 3. TSA treatment induces autophagy via blocking mTOR pathway. (A) MCF10A and MCF10A-ras cells were treated with $0.5 \mu \mathrm{M}$ TSA for $12 \mathrm{~h}$. The cells were harvested and subjected to western blotting analysis for phospho-AKT, phospho-S6, LC3, P62, Cathepsin D and HSP70 levels. $\beta$-actin was used as a loading control. (B) MCF10A and MCF10A-ras cells were treated with $0.5 \mu \mathrm{M}$ TSA for $12 \mathrm{~h}$ with or without chloroquine $(25 \mu \mathrm{M})$ for $24 \mathrm{~h}$. The cells morphology was examined under phage-contrast microscopy. Scale bar $100 \mu \mathrm{m}$. (C) MCF10A and MCF10A-ras cells were treated with $0.5 \mu \mathrm{M}$ TSA, with or without chloroquine $(25 \mu \mathrm{M})$ for $24 \mathrm{~h}$. The cell viability was measured using PI live cell uptake assay coupled with flow cytometry. Data are illustrated as the mean $\pm \mathrm{SD}(" \mathrm{P}<0.05)$. (D) Flow cytometry of MCF10A and MCF10A-ras cells were treated with $0.5 \mu \mathrm{M}$ TSA, with or without chloroquine ( $25 \mu \mathrm{M})$ for $24 \mathrm{~h}$. TSA, trichostatin A; mTOR, mammailian target of rapamycin.

Previous studies have shown that HDACIs facilitated autophagy in cancer cells $(14,22)$. Nonetheless, the role of autophagy in HDACIs-mediated cancer cell death is still controversial. Some studies have shown that autophagy served as a pro-death role through autophagy inhibition and reduction of HDACIs cytotoxicity through Atgs (autophagy associated gene) knockdown $(23,24)$. On the contrary, some other studies have revealed that autophagy served as a pro-survival mechanism $(10,25,26)$. We found that TSA reduced the expression of phospho-AKT and phospho-S6 and increased the levels of expression of LC3. These results clearly showed that TSA treatment led to autophagy in MCF10A-ras cells, which was consistent with earlier reports on HDACI-induced

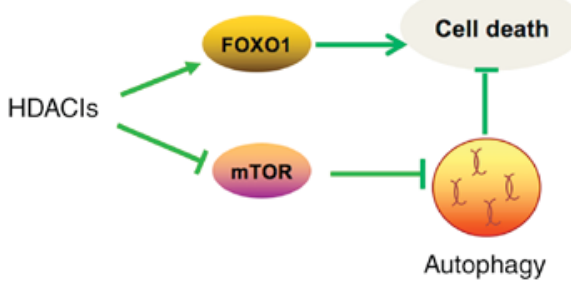

Figure 4. Illustration showed that HDACIs cause MCF10A-ras cell death through activation of FOXO1 and induce autophagy through inhibition of mTOR pathway. Autophagy induced by TSA plays a protective role in MCF10A-ras cells. Thus, the combination of HDACIs and autophagy inhibitors exert a synergistic antitumor effect. TSA, trichostatin A; HDACIs, histone deacetylase inhibitors; mTOR, mammailian target of rapamycin. 
autophagy in human cancer cells (14). Moreover, it revealed that inhibition of autophagy by CQ significantly enhanced TSA-caused cell death, suggesting that autophagy served as a cell survival mechanism in TSA-treated MCF10A-ras cells. Therefore, our results supported the notion that autophagy serves as a pro-survival mechanism. Based upon our findings, we concluded that with treatment of HDACIs in cancer cells autophagy could delay the onset of apoptosis through various mechanism including elimination of reactive oxygen species (ROS) (27). Our data support the hypothesis that combinations of HDCAIs and autophagy inhibitors might be a promising therapeutic strategy for cancer patients.

In summary (Fig. 4), TSA caused morphological changes and induced apoptosis in MCF10A-ras cells through activation of FOXO1. In addition, TSA also induced autophagy through inhibition of mTOR pathway. Moreover, inhibition of autophagy synergistically enhances the antitumor effect of TSA. Our results shed some light on developing more effective cancer therapeutic strategies by combining HDACIs and autophagy inhibitors.

\section{Acknowledgements}

This study was financially supported by the Science Technology Department of Zhejiang Province, China (grant no. 2015C33173), the Traditional Chinese Medicine Fund of Zhejiang Province, China (grant no. 2011ZA010) and the Zhejiang Provincial Natural Science Foundation of China (grant no. LQ18H280006).

\section{References}

1. Jiang P and Mizushima N: Autophagy and human diseases. Cell Res 24: 69-79, 2014.

2. Choi AM, Ryter SW and Levine B: Autophagy in human health and disease. N Engl J Med 368: 651-662, 2013.

3. Janku F, McConkey DJ, Hong DS and Kurzrock R: Autophagy as a target for anticancer therapy. Nat Rev Clin Oncol 8: 528-539, 2011.

4. Santana-Codina N, Mancias JD and Kimmelman AC: The Role of Autophagy in Cancer. Ann Rev Cancer Biol 1: 19-39, 2017.

5. Mizushima N and Komatsu M: Autophagy: Renovation of cells and tissues. Cell 147: 728-741, 2011.

6. Degenhardt K, Mathew R, Beaudoin B, Bray K, Anderson D, Chen G, Mukherjee C, Shi Y, Gélinas C, Fan Y, et al: Autophagy promotes tumor cell survival and restricts necrosis, inflammation, and tumorigenesis. Cancer Cell 10: 51-64, 2006.

7. Lum JJ, Bauer DE, Kong M, Harris MH, Li C, Lindsten T and Thompson CB: Growth factor regulation of autophagy and cell survival in the absence of apoptosis. Cell 120: 237-248, 2005.

8. Jin S and White E: Role of autophagy in cancer: Management of metabolic stress. Autophagy 3: 28-31, 2007.

9. Emanuele S, Lauricella M and Tesoriere G: Histone deacetylase inhibitors: Apoptotic effects and clinical implications (Review). Int J Oncol 33: 637-646, 2008.

10. Zhang J, Ng S, Wang J, Zhou J, Tan SH, Yang N, Lin Q, Xia D and Shen HM: Histone deacetylase inhibitors induce autophagy through FOXO1-dependent pathways. Autophagy 11: 629-642, 2015.

11. Kim HJ and Bae SC: Histone deacetylase inhibitors: Molecular mechanisms of action and clinical trials as anti-cancer drugs. Am J Transl Res 3: 166-179, 2011.
12. Hoshikawa Y, Kwon HJ, Yoshida M, Horinouchi S and Beppu T: Trichostatin A induces morphological changes and gelsolin expression by inhibiting histone deacetylase in human carcinoma cell lines. Exp Cell Res 214: 189-197, 1994.

13. Liu YL, Yang PM, Shun CT, Wu MS, Weng JR and Chen CC: Autophagy potentiates the anti-cancer effects of the histone deacetylase inhibitors in hepatocellular carcinoma. Autophagy 6: 1057-1065, 2010.

14. Gammoh N, Lam D, Puente C, Ganley I, Marks PA and Jiang X: Role of autophagy in histone deacetylase inhibitor-induced apoptotic and nonapoptotic cell death. Proc Natl Acad Sci USA 109: 6561-6565, 2012.

15. Chiao MT, Cheng WY, Yang YC, Shen CC and Ko JL: Suberoylanilide hydroxamic acid (SAHA) causes tumor growth slowdown and triggers autophagy in glioblastoma stem cells. Autophagy 9: 1509-1526, 2013.

16. Eijkelenboom A and Burgering BM: FOXOs: Signalling integrators for homeostasis maintenance. Nat Rev Mol Cell Biol 14: 83-97, 2013

17. Burgering BM: A brief introduction to FOXOlogy. Oncogene 27: 2258-2262, 2008.

18. Michaelis M, Suhan T, Michaelis UR, Beek K, Rothweiler F, Tausch L, Werz O, Eikel D, Zörnig M, Nau H, et al: Valproic acid induces extracellular signal-regulated kinase $1 / 2$ activation and inhibits apoptosis in endothelial cells. Cell Death Differ 13: 446-453, 2006.

19. Jazirehi AR: Regulation of apoptosis-associated genes by histone deacetylase inhibitors: Implications in cancer therapy. Anticancer Drugs 21: 805-813, 2010.

20. Park H, Lee YJ, Kim TH, Lee J, Yoon S, Choi WS, Myung CS and Kim HS: Effects of trichostatin A, a histone deacetylase inhibitor, on the regulation of apoptosis in H-ras-transformed breast epithelial cells. Int J Mol Med 22: 605-611, 2008.

21. Yang Y, Zhao Y, Liao W, Yang J, Wu L, Zheng Z, Yu Y, Zhou W, Li L, Feng J, et al: Acetylation of FoxO1 activates Bim expression to induce apoptosis in response to histone deacetylase inhibitor depsipeptide treatment. Neoplasia 11: 313-324, 2009.

22. Del Bufalo D, Desideri M, De Luca T, Di Martile M, Gabellini C, Monica V, Busso S, Eramo A, De Maria R, Milella M and Trisciuoglio D: Histone deacetylase inhibition synergistically enhances pemetrexed cytotoxicity through induction of apoptosis and autophagy in non-small cell lung cancer. Mol Cancer 13: 230, 2014

23. Yang PM and Chen CC: Life or death? Autophagy in anticancer therapies with statins and histone deacetylase inhibitors. Autophagy 7: 107-108, 2011.

24. Hrzenjak A, Kremser ML, Strohmeier B, Moinfar F, Zatloukal K and Denk H: SAHA induces caspase-independent, autophagic cell death of endometrial stromal sarcoma cells by influencing the mTOR pathway. J Pathol 216: 495-504, 2008

25. Carew JS, Medina EC, Esquivel JA II, Mahalingam D, Swords R, Kelly K, Zhang H, Huang P, Mita AC, Mita MM, et al: Autophagy inhibition enhances vorinostat-induced apoptosis via ubiquitinated protein accumulation. J Cell Mol Med 14: 2448-2459, 2010.

26. Lopez G, Torres K, Liu J, Hernandez B, Young E, Belousov R, Bolshakov S, Lazar AJ, Slopis JM, McCutcheon IE, et al: Autophagic survival in resistance to histone deacetylase inhibitors: Novel strategies to treat malignant peripheral nerve sheath tumors. Cancer Res 71: 185-196, 2011.

27. Ungerstedt JS, Sowa Y, Xu WS, Shao Y, Dokmanovic M, Perez G, Ngo L, Holmgren A, Jiang X and Marks PA: Role of thioredoxin in the response of normal and transformed cells to histone deacetylase inhibitors. Proc Natl Acad Sci USA 102: 673-678, 2005.

This work is licensed under a Creative Commons Attribution-NonCommercial-NoDerivatives 4.0 International (CC BY-NC-ND 4.0) License. 